\title{
High ThroughPUT OF ASYNChronOUS TRAFFIC AND MAXIMUM CAPACITY OF SYNCHRONOUS SOURCES WITH THE NEW FDDI'S MAC PROTOCOL
}

\author{
Ehab Aziz Khalil \\ Department of Computer Science \& Engineering, Faculty of Electronics Engineering, \\ Menoufiya University, Menouf-32952, EGYPT
}

\begin{abstract}
It is well known that Fiber Distributed Data Interface (FDDI) offers an excellent cost-benefit and guarantees a reliable and powerful network. It is also fact that the FDDI is suitable for synchronous traffic because of the high speed and the performance guarantees it provides. However, these guarantees are achieved at the expense of asynchronous traffic because the synchronous messages are given highest priority over asynchronous messages, resulting in excessive delays and low throughput for asynchronous message. This paper, presents a modification of the FDDI's MAC(Media Access Control) protocol to control the maximum token rotation time not to exceed the TTRT (Target Token Rotation Time) in the worst case, the realization of this condition leads to set the TTRT to the message generation period $\left(T_{g}\right)$ instead of one half of it $\left(T_{g} / 2\right)$. The new protocol of FDDI's MAC increasing both the throughput of asynchronous traffic and the capacity of synchronous sources. The proposed MAC is evaluated by means of computer simulation. The simulation results confirm that the new proposed MAC provides FDDI more efficient for supporting multimedia applications.
\end{abstract}

\section{KEYWORDS}

FDDI's MAC, Throughput of Asynchronous Traffic, Numbers of Synchronous Sources,

\section{INTRODUCTION}

The term multimedia has become one of the major buzzwords in computing and telecommunication for the current and the future. Guaranteeing service to asynchronous traffic is a problem that has been largely overlooked in the study of real time communications [1,2]. Generally, different types of packets in a computer network have different service requirements. In real-time systems, such as packetized audio communication, synchronous packets are generated periodically. Each packet generated by the source station must be received by the destination station within a specified amount of time. A certain amount of packet loss is tolerable, and a packet that is not successfully delivered within a certain time limit is considered lost [3]. However, for critical real-time systems, all synchronous packets are required to be delivered before their deadlines i.e., before the end of the period in which they arrive [4]. In other words, each synchronous packet is required to be transmitted before the generation of next one. On the other hand, there are some packets in the network, like those related to asynchronous traffic such as file transfer and e-mail, can tolerate a relatively large latency but may introduce a heavy average traffic. Although asynchronous packets can tolerate a relatively large latency, they usually require a certain guaranteed bandwidth of the network. Thus, it is practically important to address the problem of maximizing the guaranteed throughput for asynchronous packets while meeting the delivery deadlines for synchronous packets. The FDDI is the most efficient $100 \mathrm{Mbps}$ technology available today. It allows to build high speed networks that span up to $100 \mathrm{Km}$. The MAC of the FDDI is operated according to the so-called Timed Token Rotation (TTR) protocol $[5,6]$. During ring initialization process, a protocol parameter called Target Token Rotation Time (TTRT) is determined, which indicates the expected target token rotation time. Each 
station is assigned a portion of the TTRT known as Synchronous bandwidth Allocation $\left(S A_{i}\right)$, which represents the maximum permitted time for station $i$ to transmit its synchronous packets every time it receives the token. The transmission of asynchronous packets is controlled by the Token Rotation Time (TRT), which measures the time between successive token arrivals at a station. Once a station receives the token, the value of $T R T$ is copied into the Token Holding Time (THT) only if the token arrived earlier than expected. The station can then transmit its synchronous packets, if it has, within duration no more than its $S A_{i}$. Then, it transmits its asynchronous packets, if it has, until the THT expires. However, if the token is late, i.e., the TRT expires while the token rotating around the ring, synchronous packets is only served.

Our previous work $[7,8]$ confirms that the FDDI network can effectively handle synchronous transmission of both video and audio. However, a raised problem with that protocol is how to provide a maximum throughput for asynchronous traffic (such as images, graphics, text, and data). There are many related works and researches were studied different applications using the FDDI [9-57].

\section{SCOPE OF THE PROBLEM}

In general, the medium access delay of a packet is defined as the time period between its generation at a station and the beginning of its transmission. The FDDI token ring can guaranteed both the medium access delay and bandwidth for synchronous packets. The transmission delay is controlled by the ring's TTRT, which guarantees each station to have a chance to transmit its synchronous packets at least once every $2 * T T R T$ (in the worst case). It is also ensure the average time between two consecutive token's visit to a station not to exceed the TTRT [6,58-60]. The bandwidth of synchronous traffic for station $i$ to transmit is guaranteed by assigning the station $i$ a portion of the ring's TTRT, called Synchronous Allocation $\left(S A_{i}\right)$. Specifically, once station $i$ get the token, it is allowed to transmit its synchronous packets for duration up to $S A_{i}$. However, under heavy synchronous traffic, asynchronous traffic suffers from very large latency and light throughput. This problem occurs for the following reasons:

(1) The FDDI gives highest priority to synchronous traffic and asynchronous traffic is only serviced when the time permits. That is, following an opportunity to transmit synchronous packets, a station gets the opportunity to allocate any unused bandwidth to the transmission of asynchronous packet. Therefore, under heavy load of synchronous traffic, the achievable throughput of asynchronous traffic becomes light. To overcome this deficiency, it is important to take a priori assumptions about the worst case utilization of asynchronous traffic. In other words, specify a part of the bandwidth used to service asynchronous packets in the worst case.

(2) The difference between the maximum token rotation time $(2 *$ TTRT) and the average token rotation time (TTRT) reduces the FDDI token ring's capability for multimedia applications. For more detail, let $T_{\text {ring }}$ denotes the ring's latency, which is the time needed to circulate the token around the ring once without transmitting any packet, and $T_{p}$ is the time needed to transmit a maximum packet length. Since $\Sigma_{i=0} S A_{i} \leq T T R T-T_{\text {ring }}-T_{p}$, then the maximum number of synchronous sources that can be carried is $n=\left(T T R T-T_{\text {ring }}-T_{p}\right) / S A_{i}$. Suppose that the packet generation period is $T_{g}$ and the time needed to transmit a packet is $x$. Since the maximum token rotation time is $2 * T T R T$, then the TTRT must set to one half of the requested delay (i.e., $T T R T=T_{g} / 2$ ) in order to ensure that a station can get the token at least once every $T_{g}$ unit of time. To give a station enough time to transmit at least one packet after getting the token, the $S A_{i}$ of the station must be set to be not less than $x$. In that case, the maximum number of sources is $\mathrm{n}=\left(\left(T_{g} / 2\right)-T_{\text {ring }}-T_{p}\right) / \mathrm{x}$. However, if the worst case doesn't happen, the $T T R T$ can be set to $T_{g}$, and consequently improve the number of synchronous sources that can be carried on the network to be $n=\left(T_{g}-T_{\text {ring }}-T_{p}\right) / \mathrm{x}$. Thus, the difference between the maximum and the average token rotation times reduces the FDDI token ring's capability of handling synchronous traffic. Also, this difference reduces the achievable throughput of asynchronous traffic. Notice that, the $100 \mathrm{Mbps}$ transmission bandwidth of the FDDI network is always partitioned into three parts: the transmission of synchronous packets, asynchronous packets, and token passing. During one rotation of the token, the time used for token passing is constant, $T_{\text {ring. }}$. Since, the total utilization of the FDDI network is $\mathrm{U}=\left(T T R T-T_{\text {ring }}\right) / T T R T$, therefore, the faster the token rotates (i.e., small value of TTRT), the longer ring bandwidth is wasted for passing the token. In other words, for a given synchronous 
throughput, the larger an average token rotation time that a protocol can achieve, the more asynchronous packets could be transmitted (i.e., high asynchronous throughput).

\section{EFFECT OF TUNING TTRT}

In order to check the necessary to set the TTRT to one half of the requested delay $\left(T_{R e q}=T_{g}\right)$, two configuration of the FDDI token ring protocol are simulated. The first one for the TTRT equal's to one half of the requested delay (i.e., $T T R T=T_{g} / 2$ ) while the other one for the TTRT equal's to the requested delay (i.e., $T T R T=T_{g}$ ), and we study how many packets will not be transmitted before the generation of the next one.

The FDDI network is simulated with 520 stations and $92 \mathrm{Km}$ ring length. The station has a latency of $0.6 \mu \mathrm{s}$, and the propagation delay through the link is $5.085 \mu \mathrm{s} / \mathrm{Km}$. Thus, the ring has a latency $T_{\text {ring }}=$ $0.78 \mathrm{~ms}$. The synchronous traffic is considered as an audio signals with rate $\left(V_{a}\right)$ equal's to $192 \mathrm{Kbps}$. The synchronous packets are generated periodically with audio packet length $\left(P_{a}\right)$ equal's to $768 \mathrm{~B}$. Therefore, the packet generation period $\left(T_{g}\right)$ is equal to $32 \mathrm{~ms}$. Each audio packet is required to be transmitted before the generation of the next one. To simulate the worst-case, suppose all audio sources start generating audio packets at zero time. Asynchronous messages of a fixed size $M_{d}=10$ $\mathrm{KB}$ are generated with uniformly distributed interarrival time $\left(\mu_{s}\right)$, by those 20 stations that starting from station number 1 , such that the data traffic intensity $\left(\rho_{d}\right)$ is equal to 1 .

Figure 1 shows the percentage of audio packets that missing their deadlines versus number of audio sources $\left(N_{a}\right)$, for $T T R T=T_{g} / 2=16 \mathrm{~ms}$ and $T T R T=T_{g}=32 \mathrm{~ms}$. In both cases, the $S A_{i}$ of station $i$, which has audio source, is set to the time needed to transmit maximum packet length (i.e., $S A_{i}=768 \mathrm{~B} /$ $100 \mathrm{Mbps}=0.06144 \mathrm{~ms})$. Theoretically, the maximum number of audio sources $\left(N_{a-\max }\right)$ to be carried on the network is equal to 246 with $T T R T=16 \mathrm{~ms}$, and $N_{a-\max }$ equal's to 507 with $T T R T=32 \mathrm{~ms}$.

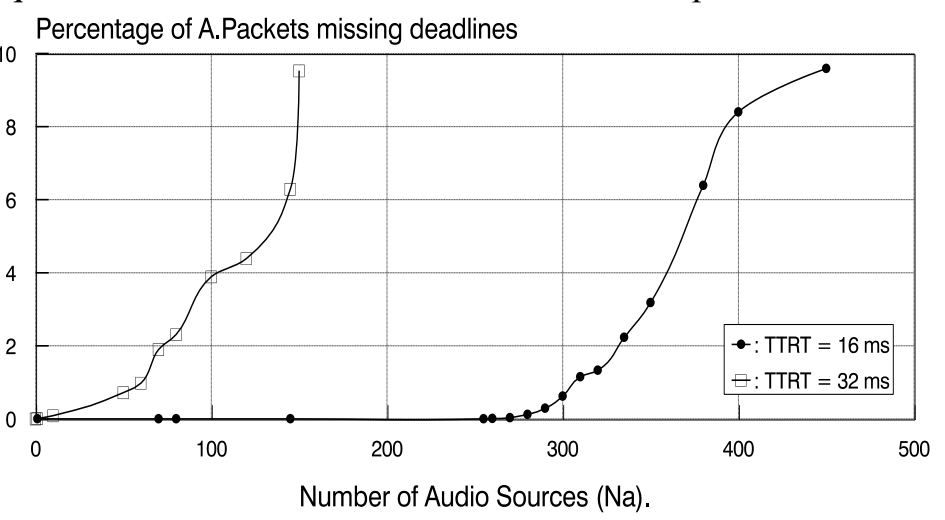

Figure 1 Percentage of Audio Packets Missing Their Deadlines Versus $\mathrm{Na}$ (FDDI).

As shown in Figure 1, up to 260 audio sources can be carried on the FDDI ring with TTRT=16 ms without any late packets. On the other hand, with TTRT $=32 \mathrm{~ms}$, the FDDI does not work at all. The audio packets missed their deadlines even in case of light number of video sources. This is because, the token rotation time could exceed the TTRT as the result of the occurrence of the worst case. Thus, in the FDDI networks, it is necessary to set the TTRT to one half of the requested bound delay.

Figure 2 shows the effect of tuning the TTRT on asynchronous throughput under the same conditions mentioned above. As shown in the Figure, given $N_{a}$, as the TTRT increases, the achievable throughput is also slightly increase. This is because, for a given $N_{a}$, the required bandwidth to synchronous packets is constant $\left(N_{a}^{*} V_{a}\right)$. Since the total utilization of the FDDI network is $U=(T T R T$ $T_{\text {ring }}$ ) / TTRT, therefore, as the TTRT increases, the asynchronous throughput increases because $T_{\text {ring }}$ is constant. From the Figure, given the TTRT, the achievable throughput decreases as $N_{a}$ increases. This is due to the increasing of the bandwidth used to service synchronous packets. Since the transmission of synchronous packets has the highest priority, then, under heavy load of synchronous packets, the achievable throughput of asynchronous traffic may reach to zero. 


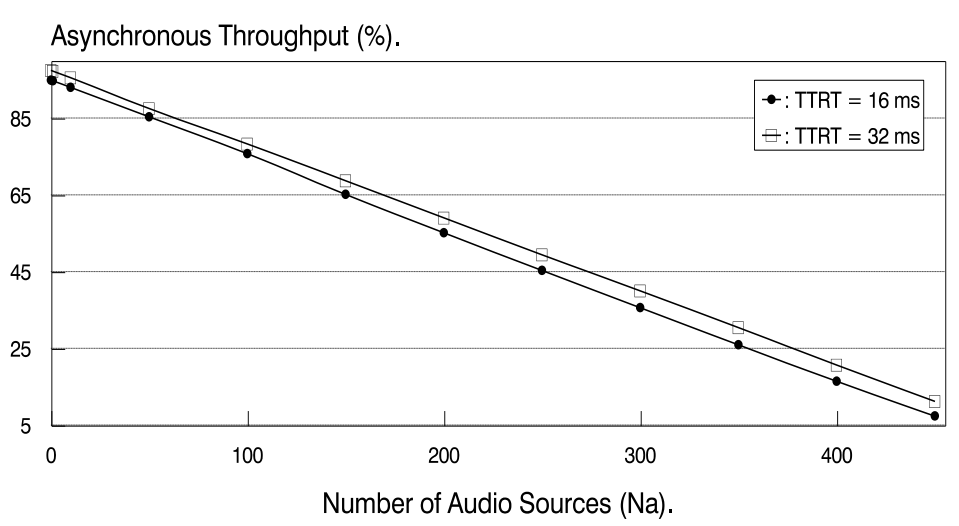

Figure 2 Asynchronous Throughput Versus $\mathrm{Na}$ (FDDI)

\section{MODIFICATIONS OF THE FDDI's MAC}

Due to the occurrence of the worst-case, it is necessary to set the TTRT to one half of the requested delay. However, as mentioned earlier, when the TTRT becomes short, long bandwidth is used for token passing. Consequently, the FDDI token ring's capability of supporting multimedia applications becomes very weak.

The idea of the FDDI's MAC protocol modifications is to control the maximum token rotation time not to exceed the TTRT, in the worst case. The realization of this condition leads to set the TTRT to the message generation period $\left(T_{g}\right)$ instead of one half of it $\left(T_{g} / 2\right)$. Consequently, maximize the guaranteed asynchronous throughput and increase the FDDI's capability of supporting synchronous traffic. It is noted that, the worst-case takes place during the second rotation that follows the ring initialization and for any other place has the following conditions: 1) no packets were transmitted during the previous token rotation; and 2) the maximum number of packets are transmitted during the current rotation. As mentioned earlier, the token rotation time is composed of three components of time: the transmission time of synchronous packets, as well as asynchronous packets, and the ring latency $\left(T_{\text {ring }}\right)$. During a cycle of the token, given the ring length and number of nodes, $T_{\text {ring }}$ is constant and the time used for transmitting synchronous packets is bounded by $\mathrm{TS}=\sum_{I=0} S A_{i}$. So if we can control the time used for transmitting asynchronous packets not to exceed $T_{A}$, such that $T_{A}+$ $T_{S}+T_{\text {ring }}=T T R T$, the maximum token rotation will never exceed TTRT. This can be realized by making the following modifications to the FDDI's MAC protocol:

(1) At ring initialization; set $L C_{i}$ (late counter) to 1 instead of 0 , in order to prevent the transmission of asynchronous packets during the second cycle of the ring's initialization, and consequently, overcome the occurrence of the worst-case in this cycle.

2) Set a new parameter called total Asynchronous Time $\left(T_{A}\right)$, represents the total time used to service asynchronous packets through a complete token's cycle, to be $T_{A} \geq\left(\alpha^{*} T T R T\right)-T_{\text {ring }}$.

This can be realized by setting $T_{A}=T T R T-T_{S}-T_{\text {ring }}$ if $T_{S} \leq(1-\alpha) T T R T$ otherwise $T A=\alpha T T R T-T_{\text {ring }}$. Where: $\alpha$ is a reserved portion of the TTRT allowed for transmitting asynchronous packets, in the worst-case, plus the time needed to circulate the token around the ring once without transmitting any packet, such that $\left(T_{\text {ring }} / T T R T\right) \leq \alpha \leq 1 / 2$.

3) Set the Token Holding Time $\left(T H T_{i}\right)$ to $T_{A}$ as long as a station start retransmitting the token after visiting it. Allow the $T H T_{i}$ to count down as long as asynchronous packets are being transmitted or forwarded by the station.

With these modifications, the token holding timer (THT) at each station ensures that: the time used by the station for transmitting its asynchronous packets plus the time used for transmitting asynchronous packets through the previous token's rotation doesn't exceed $T A=\alpha T T R T-T_{\text {ring }}$, as long as $T_{S}=\sum_{\mathrm{i}=0}$ $S A_{i} \geq(1-\alpha) T T R T$, therefore, the maximum token rotation time will never exceed $T_{A}+T_{S}+T_{\text {ring }}=T T R T$. This property removes the deficiencies of the FDDI's protocol that mentioned earlier. From now, $T T R T$ can be set to the minimum requested delay instead of one half of it. 


\section{The EFFICIENT FDDI's MAC PROTOCOL}

Suppose there are $\mathrm{N}$ stations on the ring which are numbered from 0 to $\mathrm{N}-1$. The token circulates among all the $\mathrm{N}$ stations of the ring in a round-robin fashion. The stations' access to the ring is then controlled by the following protocol:

1) As part of the FDDI ring's initialization process; each station declares TTRT to the requested transmission delay of its synchronous messages, instead of one half of it. The shortest among them is selected as the ring's TTRT. Each station supports synchronous traffic is then assigned a portion of $T T R T$ to transmit its synchronous packets. Let $S A_{I} \geq 0$ denote the portion of TTRT that assigned to station $i$.

2) Each station has two timers: Token Rotation Timer (TRT) and Token Holding Timer (THT). During the first token rotation, no packets are allowed to be transmitted and the following parameters are initialized at all stations: i) TRTI $=$ TTRT; ii) THTi $=0$; iii) $\mathrm{LCi}=1$.

3) Set the total Asynchronous Time (TA), allowed for transmitting asynchronous packets through a complete cycle of the token to be $T_{A} \geq \alpha T T R T-T_{\text {ring. }}$. This can be realized as explained earlier.

4) The $T R T_{I}$ always counts down, while the $T H T_{i}$ counts down only when a station is transmitting or forwarding asynchronous packets. When a station's THT reaches zero before the token arrives it, the token is marked as "late" by incrementing the station late counter $(\mathrm{LCi})$ by one. Meanwhile, when a station's $T R T$ reaches zero before the token arrives it, the ring recovery process is initialized.

5) The station, which has the token, is eligible to transmit packets. The packet transmission time is controlled by the timer, but a station is permitted to transmit a packet only if the transmission can be completed before the timer $T H T_{i}$ or $S A_{i}$ expires. When station $i$ receives the token, its response depends on whether the token is early or late. A token is considered to arrive early at station $i$ if $L C_{i}=0$, and late if $L C_{i}>0$ at the time of its arrival.

A) If the token arrives early, the following actions take place: i) $T R T_{i}=T T R T$; ii) If the station has synchronous packets, it transmits them either within a span of time up to $S A_{i}$ or until all the synchronous packets are transmitted, whichever occurs first; iii) The station enables $T H T_{i}$ to count down and begins transmitting asynchronous packets until $T H T_{i}$ reaches zero or all its asynchronous packets are transmitted, whichever occurs first. However, if a station doesn't use up the guaranteed synchronous bandwidth $\left(S A_{i}\right)$, the remaining bandwidth can be used to transmit asynchronous packets; iv) $T H T_{i}$ is reset to $T_{A}$ in order to compute the total time used to service asynchronous packets during the next rotation up to receive the token.

B) When the token arrives late, the following actions take place: i) $T R T_{i}=T T R T$; ii) $L C_{i}=0$; iii) Station $i$ can transmit synchronous packets (if any) for either a maximum time of $S A_{i}$ or until all the synchronous packets are transmitted, whichever occurs first; iv) No asynchronous packets will be transmitted; v) $T H T_{i}$ is set to $T_{A}$ (i.e., $T H T_{i}=T_{A}$ ).

6) Station i passes the token to next station $(i+1)$.

\section{RESULTS AND DISCUSSION}

To verify the advantages of the modified FDDI over the standard FDDI protocol for supporting multimedia applications, an extensive simulation is carried out. The purpose of this simulation is to locate the achievable throughput of asynchronous traffic and the maximum capacity of synchronous sources that can be carried with the two protocols. The FDDI's MAC protocol, the synchronous traffic, and the asynchronous traffic are simulated by the same assumptions mentioned in section 3 . In addition to that, two configurations of the modified FDDI's MAC protocol are simulated, the first one with $\alpha=1 / 2$, and the second with $\alpha=1 / 4$. The first configuration is used to confirm that, the capability of the modified FDDI is the same as the FDDI for supporting synchronous traffic, however, it achieves higher asynchronous throughput. While the second configuration is used to confirm that the modified FDDI provides higher performance than the standard FDDI for supporting both traffics. Ideally, the 100 Mbps token ring with audio coding rate $\left(V_{a}\right)$ of $192 \mathrm{Kbps}$ can support 520 audio sources. However, in order to ensure that each audio packet to be transmitted before the generation of next one: (i) the token must visit a station at least once every $T_{g}$; and (ii) the guaranteed bandwidth for each audio source must equal to the time needed to transmit a maximum-size packet. For the standard FDDI, the TTRT must set to $T_{g} / 2=16 \mathrm{~ms}$, and the $S A_{i}$ of a station that generates audio packets must 
International Journal of Engineering Sciences \& Emerging Technologies, Feb 2012.

ISSN: 2231 - 6604 doi: 10.7323/ijeset/v1_i2_4 Volume 1, Issue 2, pp: 27-36 CIJESET

set to $(768 \mathrm{~B} / 100 \mathrm{Mbps}) 0.06144 \mathrm{~ms}$. In that case, no more than 246 audio sources could be supported. On the other hand, with the modified FDDI, TTRT is set to the packet generation period of $32 \mathrm{~ms}$ and $S A_{i}$ is set to $0.06144 \mathrm{~ms}$. Then, no more than 260 audio sources can be carried with $\alpha=$ $1 / 2$, while no more than 390 audio sources could be carried with $\alpha=1 / 4$.

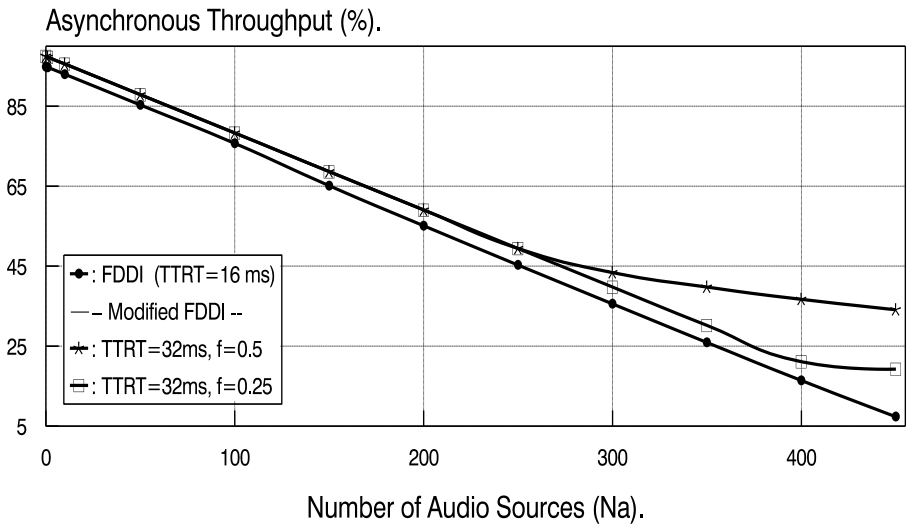

Figure 3 Asynchronous Throughput Versus $\mathrm{Na}$ (FDDI \& Modified FDDI)

Figure 3 illustrates the throughput characteristics of asynchronous traffic for the standard FDDI and the modified FDDI protocols. The Figure shows that, the achievable throughput of the modified FDDI protocol is higher than that of the standard FDDI protocol. However, with the modified FDDI protocol, as $\alpha$ increases, the achievable throughput is also increase. This is because the reserved portion of the TTRT for transmitting asynchronous packets increases as $\alpha$ increases. This performance can be seen more clearly in Figure 4, which shows the average token rotation time for the standard FDDI and the modified FDDI protocols under the same conditions of Figure 3. As mentioned earlier, the shorter average token rotation time, the longer transmission bandwidth that used for the token passing, thus leaving less bandwidth for asynchronous traffic. The difference between asynchronous throughput is more pronounced with the increase of the average token rotation time.

Figure 5 shows the percentage of audio packets that missing their deadlines as a function of the number of audio sources $\left(N_{a}\right)$, for the standard FDDI and the modified FDDI. From the figure, it is clear that up to 260 audio sources can be carried by the FDDI network without any late of audio packets. However, setting up any more audio sources beyond 260 causes the audio packets to be missed their deadlines.

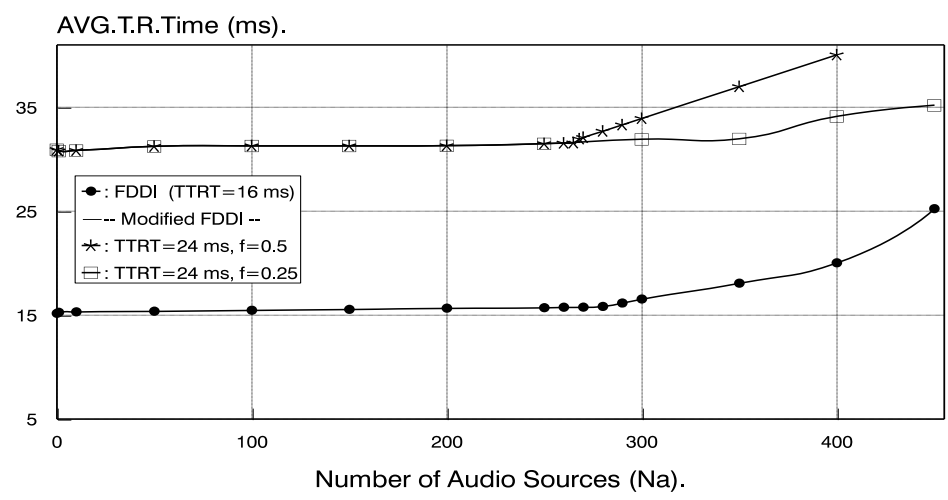

Figure 4 Average Token Rotation Time (FDDI \& FDDI- M) 


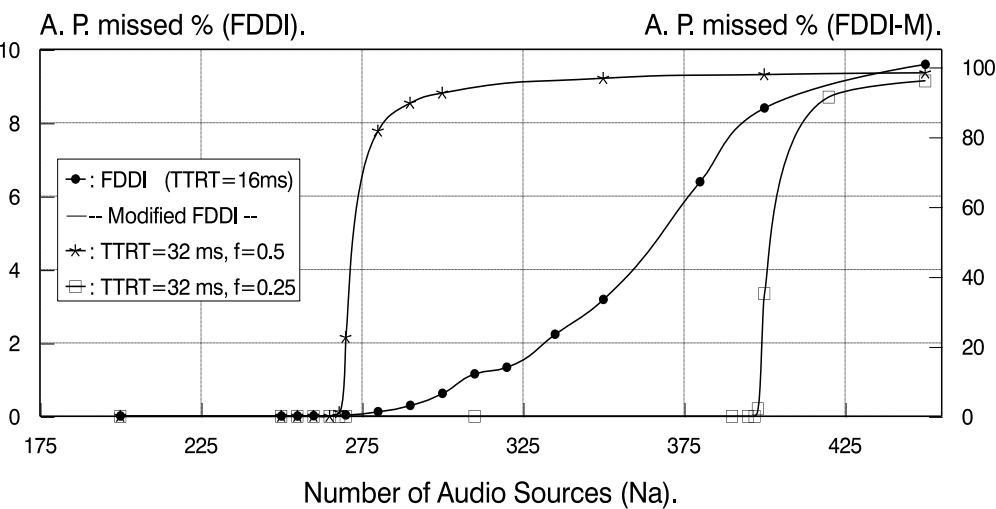

Figure 5 Percentage of Audio Packets Missing Their Deadlines Vs $N a$ (FDDI and modified FDDI).

On the other hand, the modified FDDI behaves exactly as estimated, up to 260 audio sources can be carried with $\alpha=1 / 2$, and up to 390 audio sources can be carried with $\alpha=1 / 4$. As expected, the modified FDDI outperforms the standard FDDI by far with $\alpha=1 / 4$, it can accommodate up to 130 audio sources more than the standard FDDI. In addition to that, it achieves higher throughput than the standard FDDI as shown in Figure 3. Also, Figure 5 shows that, when $\alpha=1 / 2$, the capability of the modified FDDI for supporting audio sources is the same as the standard FDDI. However, it can achieve higher throughput than the standard FDDI as shown in Figure 3.

\section{CONCLUSION}

Looking just at the high throughput of asynchronous traffic and the maximum capacity of the audio sources can be carried, the proposed FDDI's MAC protocol appears superior. The main advantage of the proposed protocol are guaranteed high throughput for asynchronous massages and increase the number of audio sources. We have explained the FDDI's parameters which have the effect to maximize the guaranteed throughput for asynchronous massages, while still providing the same guarantee to synchronous message. The simulation results confirm that the proposed protocol improves the FDDI's efficiency for supporting multimedia applications. This reflects in the increasingly strong position of FDDI to form the core of an efficient architecture for integrated services. The future work will focus on the generalization of the model to include different types of time constrained packets and applications to real life problems.

\section{REFERENCES}

[1] M. Hamadaoui, and P. Ramanathan (1995), "Deferring Real Time Traffic for Improved Non-Real Time Communication in FDDI Networks," IEEE Trans. on Computers, Vol. 44, No. 12, Dec. 1995, pp. 1420 1428.

[2] K. G. Shin, Q. Zheng (1993), "Mixed Time-Constrained and Non-Time Constrained Communications in Local Area Networks," IEEE Trans. Commun., Vol. 41, No. 11, Nov. 1993, PP. 1668-1676.

[3] Francois Fluckiger (1995), "Understanding Networked Multimedia Applications and Technology," Prentice Hall, 1995.

[4] G. Agrawal, B. Chen, W. Zhao, and S. Davari (1994), "Guaranteeing Synchronous Message Deadlines with The Timed Token Medium Access Control Protocol," IEEE Trans. Comput., Vol. 43, No. 3, March 1994.

[5] P. Davids, T. Meuser, and O. Spaniol (1994), "FDDI: Status and Perspectives," Computer Networks and ISDN Systems 26, 1994, pp. 657-677.

[6] C.M. Bakes, and F.N. Goldberg (1995), “Applications of Fibre Optic Networks in High Technology Research," Int. J. of Computer Applications in Technology, Vol. 8, Nos 3/4, 1995, pp. 172-189.

[7] E. A. Khalil, and G. M. Ali (1998), "Performance Evaluation of FDDI High Speed LAN for Supporting Multimedia Applications," The Twenty Third International Conference for Statistics, Computer Science and Its Applications, EGYPT, May 1998. 
[8] G. M. Ali, E. A. Khalil (1999), "Applications of Multimedia in High Speed Networks" M. Sc Thesis, Dept. of Computer Science \& Engineering, Faculty of Electronic Engineering, EGYPT, 1999.

[9] A. P. Jayasumana and P. N. Werahera (1990), "Performance of Fibre Distributed Data Interface Network for Multiple Classes of Traffic," IEEE Proc., pt. E, vol. 137, no. 5, Sept. 1990, pp. 401-408.

[10] Wang, J. Zhang, S. Maple, C. (2011), " Guaranteeing the Timely Transmission of Periodic Messages with Arbitrary Deadline Constraints Using the Timed Token Media Access Control Protocol ," In the Communications, IET, Volume: 5 Issue: 4, March 2011 , pp. 519 - 533.

[11] Jun Wang, Sijing Zhang, Carsten Maple, Zhengxu Zhao (2009), "Guaranteeing Hard Real-Time Traffic with Legitimately Short Deadlines with the Timed Token Protocol," In the Journal of Computer Standards \& Interfaces , Volume 31 Issue 3, March 2009.

[12] Sijing Zhang, Alan Burns, Jing Chen, E. Stewart Lee (2004), "Hard Real-Time Communication with the Timed Token Protocol: Current State and Challenging Problems," Published in: · Journal Real-Time Systems, Volume 27 Issue 3, September 2004.

[13] Sijing Zhang, Alan Burns, Tee-Hiang Cheng (2002), " Cycle-Time Properties of the Timed Token Medium Access Control Protocol," Journal IEEE Transactions on Computers, Volume 51 Issue 11, November 2002.

[14] Sijing Zhang, Alan Burns, Ahmed Mehaoua, E. Stewart Lee, Hongji Yang (2002), " Testing the Schedulability of Synchronous Traffic for the Timed Token Medium Access Control Protocol," Journal RealTime Systems, Volume 22 Issue 3, May 2002.

[15] Ching-Chih Han; Shin, K.G.; Chao-Ju Hou (2001), " Synchronous Bandwidth Allocation for Real-Time Communications with the Timed-Token MAC Protocol," IEEE Transactions on Computers, Volume: 50 Issue:5, May 2001, pp. $414-431$.

[16] Sijing Zhang, E. Stewart Lee (1999), " Efficient Global Allocation of Synchronous Bandwidths for Hard Real-Time Communication with the Timed Token MAC Protocol," In the Proceeding of RTCSA '99 Proceedings of the Sixth International Conference on Real-Time Computing Systems and Applications, December 1999.

[17] Sijing Zhang, E. Stewart Lee (1999), " The Worst-Case Scenario for Transmission of Synchronous Traffic in an FDDI Network," In the Proceeding LCN '99 Proceedings of the 24th Annual IEEE Conference on Local Computer Networks, October 1999.

[18] D. Chen, V.C.S. Lee, E. Chan (1998), " On the Ability of the FDDI-M Protocol to Support Real-Time Traffic," In the Fifth International Conference on Real-Time Computing Systems and Applications (RTCSA'98), Hiroshima, Japan, October 27-29, 1998, Page 51.

[19] Sijing Zhang, Alan Burns (1997), "Timing Properties of the Timed Token MAC Protocol," In the Proceeding of IC3N '97 Proceedings of the 6th International Conference on Computer Communications and Networks, September 1997, IC3N '97.

[20] Sijing Zhang, Tee-Hiang Cheng, K. R. Subramanian, Liren Zhang, Chee Heng Tan (1997), "Testing the Feasibility of Synchronous Bandwidth Allocation for Time-Critical Communication in FDDI Networks," Proceeding of the IC3N '97 Proceedings of the 6th International Conference on Computer Communications and Networks, September 1997, IC3N '97.

[21] Y Ofek, K Sohraby, Ho-Ting Wu (1997), "Integration of Synchronous and Asynchronous Traffic on the MetaRing and its Performance Study," In the IEEEACM Transactions on Networking, 1997, Vol.5, Issue 1, pp. 111-121.

[22] Sijing Zhang, Tee-Hiang Cheng, Hongji Yangt, K.R. Subramanian, Chee Heng Tan and Liren Zhang (1997), "Improving the Normalized Proportional Allocation Scheme for Time-Critical Communication in FDDI Networks," International Conference on Information, Communications and Signal Processing ICICS '97 Singapore, 9-12 September 1997, pp. 1436-1440.

[23] Sijing Zhang, Alan Burns, Andy Wellings (1996), " An Efficient and Practical Local Synchronous Bandwidth Allocation Scheme for the Timed-Token MAC Protocol," In the Proceeding of the INFOCOM'96 Proc. of the 15th annual joint conference of the IEEE computer and communications societies conference on The conference on computer commun. - Vol. 2, March 1996 INFOCOM'96.

[24] Sijing Zhang and Alan Burns (1995), "An Optimal Synchronous Bandwidth Allocation Scheme for Guaranteeing Synchronous Message Deadlines with the Timed-Token MAC Protocol ," IEEWACM TRANSA IONS ON NETWORKING, Vol. 3. No. 6. December 1995, pp. 729-741.

[25] Shin, K.G. Qin Zhen (1995), "FDDI-M: a Scheme to Double FDDI's Ability of Supporting Traffic," Parallel and Distributed Systems, IEEE Transactions on Nov 1995, Issue 11, pp. 1125-1131.

[26] Nicholas Malcolm and Wei Zhao (1994), "The Timed-Token Protocol for Real-Time Communications," In the IEEE Computer, January 1994, pp.35-41.

[27] G. Agrawal, B. Chen, B. Chen, S. Davari (1994), " Guaranteeing Synchronous Message Deadlines with the Timed Token Medium Access Control Protocol ," In the IEEE TRANSACTIONS ON COMPUTERS, vol. 43 no. 3, March 1994, pp. 327-339. 


\section{International Journal of Engineering Sciences \& Emerging Technologies, Feb 2012. ISSN: 2231 - 6604 doi: 10.7323/ijeset/v1_i2_4 Volume 1, Issue 2, pp: 27-36 CIJESET}

[28] Chug-Ju Chang, AND Hsin-Huei wu (1993), "Analysis of Synchronous and Asynchronous Traffic in an FDDI Network," TENCON '93. Proceedings. Computer, Communication, Control and Power Engineering.1993 IEEE Region 10 Conference on 19-21 Oct 1993, IEEE TENCON '93 / Beijing, China, 1993, Vol.3, pp. 187-189.

[29] Qin Zheng, Kang G. Shin (1993), "Synchronous Bandwidth Allocation in FDDI Networks," TR93-06 December 1993, MITSUBISHI ELECTRIC RESEARCH LABORATORIES, Cambridge Research Center, http://www.merl.com.

[30] B. Chen, G. Agrawal, and W. Zhao (1992), "Optimal Synchronous Capacity Allocation for Hard Real Time Communications With the Timed Token Protocol," in Proc. Real-Time Systems Symposium. IEEE, Dec. 1992.

[31] G. Agrawal, B. Chen, W. Zhao and S. Davari (1992), "Guaranteeing Synchronous Message Deadlines with Timed Token Medium Access Control Protocol," Tech. Rep. 92-006, Comput. Sci. Dept., Texas A\&M Univ., Apr. 1992.

[32] Willebeek-LeMair, M.; Schaffa, F.; Patel, B. (1992), " Isochronous versus Synchronous Traffic in FDDI," In the Proc.., of 17th Conference of Local Computer Networks, 13-16 Sep. 1992, pp. 100 - 109.

[33] Raj Jain (1991), "Performance Analysis of FDDI Token Ring Networks: Effects of Parameters and Guidelines for Setting TTRT," In the IEEE, Letter, May 1991, pp.16-22.

[34] M. Tan emann and K. Sauer (1991), "Performance Analysis of the Timed Token Protocol of FDDI and FDDI-II," IEEE Globelcom, 1991, pp. 271-278.

[35] Y. Y. Yang and R. Sankar (1991), "Maximizing FDDI Network Performance by Parameter Tuning," IlEE Globecom., 1991, pp. 1431-1439.

[36] H. Takagi (1990), "Effects of the Target Token Rotation Time on the Performance of a Timed-Token Protocol," in Proc. Performance '90, Edinburgh, Scotland. Sept. 1990, pp.363-370.

[37] Amarvir Singh (2011),"Throughput Analysis of Ethernet and Fiber Distributed Data Interface Using OPNET IT Guru Academic Edition 9.1," Research Cell: An International Journal of Engineering Sciences ISSN: 2229-6913 Issue Sept 2011,Vol. 4. pp.455-461.

[38] M A Al-Shargabi, A S Ismail (2008), "Utilizing Optical Burst Switching Networks for the Internet RealTime traffic," 2008 International Symposium on High Capacity Optical Networks and Enabling Technologies HONET 2008 (2008), pp.63-68.

[39] Ping Wang, Weihua Zhuang (2008), "A Token-Based Scheduling Scheme for WLANs Supporting Voice/Data Traffic and its Performance Analysis," IEEE Transactions on Wireless Communications (2008) Vol.7, Issue 5, Publisher: IEEE, pp.1708-1718.

[40] J Wyatt, D Habibi, I Ahmad, H Zen (2007), "Providing QoS for Symmetrical Voice/Video Traffic in Wireless Networks ," ICON 2007 Proceedings of the 2007 15th IEEE International Conference on Networks (2007), pp.312-317.

[41] Ping Du, Shunji Abe (2006), " Burst Assembly Method with Traffic Shaping for the Optical Burst Switching Network ," IEEE Globecom (2006).

[42] Ruyan Wang, Dapeng Wu, Keping Long, Weile Zhu (2006), "A Low-Latency Burst Assembly Technique in Optical Burst Switching Networks," 2006 International Conference on Communications Circuits and Systems (2006) Volume: 00, Publisher: IEEE, pp.1865-1868.

[43] L.Pushparani Devi, S. Birendra Singh and Sanasam Bimol, (2005), "A new Analysis of System Utilization Performance for Ethernet Networking System”, IEEE 802.11e EDCA, IEEE Transactions on Comm., 2005.

[44] George N Rouskas Teng Jing (2005), "Traffic Engineering Approach to Path Selection in Optical Burst Switching Networks ," Journal of Optical Networking (2005), Vol.4, Issue 11, pp.759-777.

[45] Y. Y. Yang and R. Sankar (2004), "Performance Analysis of FDDI", IEEE Network, September 2004.

[46] William J. Cronin, Jerry D. Hutchison, K.K. Ramakrishnan and Henry Yang (2003), “A comparison of High Speed LANs," Proc. of IEEE Inte'l Symposium on Network Computing and Applications, 2003.

[47] Jiangtao Luo, Qingji Zeng, Hao Chi, Zhizhong Zhang, Huandong Zhao, (2003), "The Impacts of Burst Assembly on the Traffic Properties in Optical Burst Switching Networks," Inte'l Conference on Comm. Technology Proceedings 2003 ICCT 2003 (2003), Issue 69990540, pp. 521-524.

[48] S Dharmaraja, A S Alfa, (2003),"Performance Analysis of 3G Wireless Networks Supporting Voice, Data and Video Traffic," Proc. of The Inte'l Conference On Wireless Networks 2003, pp.495-498.

[49] Shriram Patwardhan (2002), "High Speed Optical Networking with Ethernet", proceedings of 24th conference on Local Computer Networks (LCN'02), 2002.

[50] Timothy J King, "The fiber distributed data interface (FDDI) - A New Generation Standard for Local Area Networks", http://www.citeseerx.edu/

[51] Joseph Kee-Yin Ng, Victor Chung-sing Lee, (2000) "Performance Evaluation of Transmission Schemes for Real-Time Traffic in a High-Speed Timed-Token MAC Network ," Journal of Systems and Software (2000), Vol. 54, Issue 1, pp.41-60. 


\section{International Journal of Engineering Sciences \& Emerging Technologies, Feb 2012. ISSN: 2231 - 6604 doi: 10.7323/ijeset/v1_i2_4 Volume 1, Issue 2, pp: 27-36 OIJESET}

[52] Nazy Alborz, Maryam Keyvani, Milan Nikolic, and Ljiljana Trajkovic, "Simulation of Packet Data Networks Using OPNET", http:// www.citeseerx.edu.

[53] G. Bucci, A. Del Bimbo and S. Santini, (1998) "Design and Performance Evaluation of High Throughput Ethernet/FDDI Interconnect", Proceedings of Seventh International Conference on Computer Communications and Networks (ICCCN '98), 1998.

[54] S Kamat, Wei Zhao, (1996) "An Efficient Optimal Reconfiguration Algorithm for FDDI-Based Networks, " IEEE Transactions on Parallel and Distributed Systems (1996) Apr 1996, Vol.7, Issue 4, pp.411-424

[55] G Bucci, A Del Bimbo, S Santini, (1994), "Performance Analysis of two Different Algorithms for EthernetFDDI Interconnection ," IEEE Transactions on Parallel and Distributed Systems (1994), Vol.5, Issue 6, pp.614-629.

[56] Sanjay Kamat, Nicholas Malcolm, Wei Zhao, (1993), "Performance Evaluation of a Bandwidth Allocation Scheme for Guaranteeing Synchronous Messages with Arbitrary Deadlines in an FDDI Network ," IEEE Real-Time Systems Symposium, 1993, pp.34-43.

[57] Th Welzel, Peter Martini, S Rudloff, (1990) " Performance Analysis of FDDI and Multiple Token Ring Backbones in a Mixed Traffic Environment," High Speed Local Area Networks II (1990), pp.53-68.

[58] K. G. Shin, and Q. Zheng (1995), "FDDI-M: A Scheme to Double FDDI'S Ability of Supporting Synchronous Traffic," IEEE Trans. on Prallal and Dist. Systems, Vol. 6, No. 11, Nov. 1995.

[59] B. Albert, and A. P. Jayasumana (1996), "The Effects of Data Locality on FDDI-based Schemes at Gigabit Speeds," From LCN,21, 1996.

[60] B. Albert and A. P. Jayasumana (1995), "Performance Analysis of FDDI LANs using Numerical Methods," From LCN.20, 1995.

Ehab Aziz Khalil, (B.Sc'78 - M.Sc.'83 - Ph.D.'94), Ph.D. in Computer Network and Multimedia in the Dept. of Computer Science \& Engineering, Indian Institute of Technology (IIT) Bombay-400076, India in July 1994, Research Scholar from 1988-1994 with the Dept. of Computer Science \& Engineering, Indian Institute of Technology (IIT) Bombay-400076, India, M.Sc in the Systems and Automatic Control, Faculty of Electronic Engineering, Minufiya University, Menouf - 32952, EGYPT, Oct. 1983, B.Sc. in the Dept. of Industrial Electronics, Faculty of Electronic Engineering, Minufiya University, Menouf - 32952, EGYPT, May 1978. Since July 1994 up to now, working as

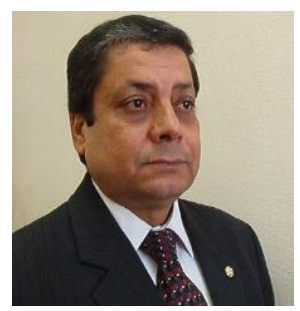
a Lecturer, with the Dept. of Computer Science \& Engineering, Faculty of Electronic Engineering, Minufiya University, Menouf - 32952, EGYPT.. Participated with the TPC of the IASTED Conference, Jordan in March 1998, and With the TPC of IEEE IC3N, USA, from 2000-2002. Consulting Editor with the "Who's Who?" in 2003-2004. Member with the IEC since 1999. Member with the Internet2 group. Manager of the Information and Link Network of Minufiya University, Manager of the Information and Communication Technology Project (ICTP) which is currently implementing in Arab Republic of EGYPT, Ministry of Higher Education and the World Bank. Published more than 87 research papers and article reviews in the international conferences, Journals and local newsletter. 